\title{
ChemComm
}

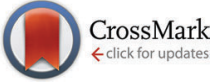

Cite this: Chem. Commun., 2015, 51, 8319

Received 23rd February 2015, Accepted 1st April 2015

DOI: $10.1039 / c 5 c c 01613 d$

www.rsc.org/chemcomm

\section{Detection of NAD(P)H-dependent enzyme activity with dynamic luminescence quenching of terbium complexes $\dagger$}

\author{
Hiroki Ito, ${ }^{\mathrm{ab}}$ Takuya Terai, ${ }^{\mathrm{ab}}$ Kenjiro Hanaoka, ${ }^{\mathrm{a}}$ Tasuku Ueno, ${ }^{\mathrm{ab}}$ Toru Komatsu, ${ }^{\mathrm{ac}}$ \\ Tetsuo Nagano ${ }^{d}$ and Yasuteru Urano*abe
}

\begin{abstract}
We discovered that positively charged terbium complexes bearing 1,4,7,10-tetraazacyclododecane functionalized with amide ligands are highly sensitive to dynamic luminescence quenching by $\mathrm{NAD}(\mathrm{P}) \mathrm{H}$. We exploited this phenomenon to establish a general time-resolved luminescence-based assay platform for sensitive detection of $\mathrm{NAD}(\mathrm{P}) \mathrm{H}$-dependent enzyme activities.
\end{abstract}

Reduced nicotinamide adenine dinucleotide (phosphate), or $\mathrm{NAD}(\mathrm{P}) \mathrm{H}$, is an important cofactor of oxidoreductases, which transfer electrons from $\mathrm{NAD}(\mathrm{P}) \mathrm{H}$ to their substrates, affording $\operatorname{NAD}(\mathrm{P})^{+}$and the products. These enzymes function in many metabolic pathways, and are considered potential therapeutic targets. ${ }^{1,2}$ Assay of their activities is also helpful for clinical diagnosis. ${ }^{3}$ However, current assays of $\mathrm{NAD}(\mathrm{P}) \mathrm{H}$-dependent enzyme activities are mostly based on absorption measurement, and this strategy has many drawbacks. For example, monitoring the absorption of $\mathrm{NAD}(\mathrm{P}) \mathrm{H}$ itself at $340 \mathrm{~nm}$, which is a common approach, gives poor sensitivity in small volumes, such as 384- or 1536-well microplates, ${ }^{4}$ and absorbance in the UV region is influenced by many extraneous factors. Colouring of tetrazolium salts upon $\mathrm{NAD}(\mathrm{P}) \mathrm{H}-$ dependent reduction is also used in cell proliferation and toxicity assays, but chemical instability of these reagents ${ }^{4}$ limits their applicability. Fluorescence measurement

\footnotetext{
${ }^{a}$ Graduate School of Pharmaceutical Sciences, The University of Tokyo,

7-3-1 Hongo, Bunkyo-ku, Tokyo 113-0033, Japan

${ }^{b}$ CREST, JST, 7 Gobancho, Chiyoda-ku, Tokyo 102-0076, Japan

${ }^{c}$ PRESTO, JST, 4-1-8 Honcho, Kawaguchi, Saitama 332-0012, Japan

${ }^{d}$ Open Innovation Center for Drug Discovery, The University of Tokyo, 7-3-1 Hongo, Bunkyo-ku, Tokyo 113-0033, Japan

${ }^{e}$ Graduate School of Medicine, The University of Tokyo, 7-3-1 Hongo,

Bunkyo-ku, Tokyo 113-o033, Japan. E-mail: t-terai@mol.f.u-tokyo.ac.jp, uranokun@m.u-tokyo.ac.jp

$\dagger$ Electronic supplementary information (ESI) available: Experimental procedures, synthetic protocols and characterization of compounds, photophysical properties of complexes, absorption and luminescence spectra of complexes upon addition of NADH, the effect of analogous compounds, an energy diagram of $\mathrm{Tb}^{3+}$ complexes, fluorescence spectra of the antenna moiety, additional data of LDH assay, detection of other enzymes, and the reversible nature of the method. See DOI: $10.1039 / \mathrm{c} 5 \mathrm{cc} 01613 \mathrm{~d}$
}

of $\mathrm{NAD}(\mathrm{P}) \mathrm{H}$ at $460 \mathrm{~nm}$ is another option, but is rarely used in practice, partly due to poor sensitivity at low $\mathrm{NAD}(\mathrm{P}) \mathrm{H}$ concentrations and an inner filter effect at high $\mathrm{NAD}(\mathrm{P}) \mathrm{H}$ concentrations. ${ }^{4}$ Although a few chemodosimeters for NAD(P)H have been reported, ${ }^{5}$ they are not suitable for monitoring enzyme activity in real time. Hence, we thought that a novel strategy to detect $\mathrm{NAD}(\mathrm{P}) \mathrm{H}$ that overcomes these disadvantages would have many potential applications, especially for high-throughput screening (HTS) in drug discovery and for clinical diagnostic tests.

For this purpose, we focused on luminescent lanthanide complexes. Lanthanide $\left(\mathrm{Tb}^{3+}\right.$ or $\left.\mathrm{Eu}^{3+}\right)$ complexes have long luminescence lifetimes of millisecond order, ${ }^{6}$ which enables sensitive detection by measuring only the long-lived component of luminescence (i.e. time-resolved measurement). By exploiting this capability, functional luminescent probes for various analytes including ions $^{7}$ and enzymes ${ }^{8}$ have been developed, ${ }^{9}$ but no luminescent probes for $\mathrm{NAD}(\mathrm{P}) \mathrm{H}$ have yet been reported. Interestingly, Parker et al. recently showed that some lanthanide complexes are susceptible to dynamic quenching by a few biological reductants such as ascorbate and urate, probably via exciplex formation. ${ }^{10,11}$ Because they were interested in the use of luminescent complexes in cells, they regarded this quenching as undesirable, with one exception, ${ }^{12}$ and they developed complexes that were tolerant of reductants. ${ }^{10}$ However, we thought that we could make use of this kind of intermolecular quenching to develop a novel and general strategy to detect $\mathrm{NAD}(\mathrm{P}) \mathrm{H}-$ dependent enzyme activity. In principle, this should be feasible, because $\mathrm{NAD}(\mathrm{P}) \mathrm{H}$ is one of the most potent biological reductants (Table S1, ESI $\dagger$ ), which would efficiently quench luminescence, whereas $\operatorname{NAD}(\mathrm{P})^{+}$, an oxidized form of $\mathrm{NAD}(\mathrm{P}) \mathrm{H}$, would not. Herein, we show that $\mathrm{Tb}^{3+}$ complexes are indeed dynamically quenched by $\mathrm{NAD}(\mathrm{P}) \mathrm{H}$ and the magnitude of the quenching can be modulated by adjusting the overall charge of the complex (Fig. 1a). Based on this finding, we have developed a novel general luminescence intensity- and lifetime-based assay for $\mathrm{NAD}(\mathrm{P}) \mathrm{H}$-dependent enzyme activities.

We first hypothesized that the efficiency of the expected quenching by $\mathrm{NAD}(\mathrm{P}) \mathrm{H}$ would depend on the structure of the 
a

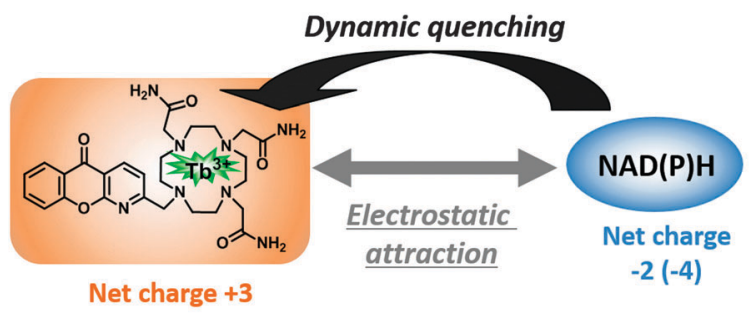

b

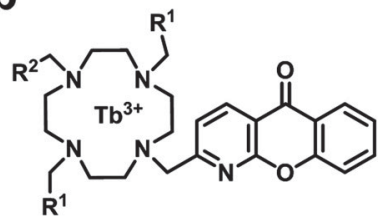

A1Tb: $\mathrm{R}^{1}, \mathrm{R}^{2}=\mathrm{CONH}_{2}$ A2Tb: $\mathrm{R}^{1}=\mathrm{COO}^{-}, \mathrm{R}^{2}=\mathrm{CONH}_{2}$ A3Tb: $R^{1}, R^{2}=\mathrm{COO}^{-}$

Fig. 1 (a) Schematic representation of the interaction of the $\mathrm{Tb}^{3+}$ complex and $\mathrm{NAD}(\mathrm{P}) \mathrm{H}$. (b) Chemical structure of complexes.

chelating moiety, in particular its charge. To test this idea, we designed and synthesized three $\mathrm{Tb}^{3+}$ complexes with different net charges (Fig. 1b). Negatively charged $\mathrm{NAD}(\mathrm{P}) \mathrm{H}$ is expected to show greater electrostatic attraction towards a more positively charged complex, as previously reported for ATP..$^{13}$ As a scaffold complex, we selected $\mathrm{A} 3 \mathrm{~Tb}$, previously reported by Parker et al., ${ }^{11 b, 12}$ and prepared a series of side-chain derivatives with altered net charge, because 1,4,7,10-tetraazacyclododecane and its derivatives form kinetically and thermodynamically stable complexes with lanthanide ions. ${ }^{14}$ Three of the ring nitrogen atoms were substituted with amide and/or carboxylate groups that coordinate to $\mathrm{Tb}^{3+}$. Azaxanthone was used as an antenna moiety because it sensitizes $\mathrm{Tb}^{3+}$ with high efficiency. ${ }^{15}$ The changes in luminescence intensity and lifetime of A1Tb, the complex with the highest positive charge, upon titration of NADH are illustrated in Fig. 2. Notably, the values of both parameters were greatly decreased in the presence of as little as $1 \mu \mathrm{M} \mathrm{NADH}$, and the addition of $100 \mu \mathrm{M} \mathrm{NADH}$ almost totally quenched the luminescence. The quenching was concentrationdependent, and the Stern-Volmer constants $\left(K_{\text {sv }}\right)$ of luminescence intensity and lifetime were almost identical at low NADH concentrations (Fig. S1, ESI $\dagger$ ), indicating that the quenching process primarily occurs in the excited state of the $\mathrm{Tb}^{3+} .{ }^{16} \mathrm{The}$ data demonstrate that $\mathrm{Tb}^{3+}$ complexes with appropriate structures are indeed dynamically quenched by NADH with high efficiency. On the other hand, complexes with less positive charge and neutral charge, $\mathrm{A} 2 \mathrm{~Tb}$ and $\mathrm{A} 3 \mathrm{~Tb}$, respectively, showed
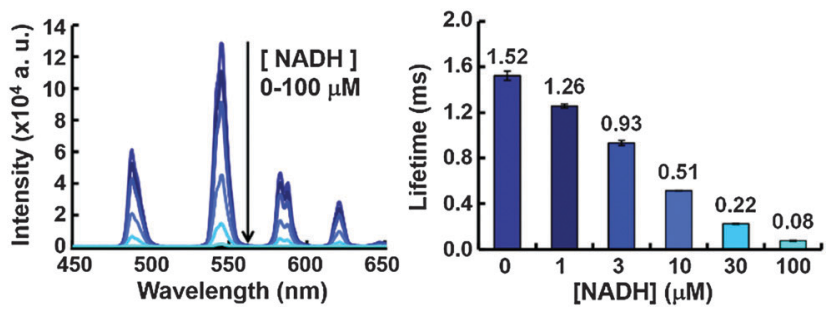

Fig. 2 Luminescence spectra (left) and luminescence lifetime (right) of A1Tb upon titration of NADH from $0 \mu \mathrm{M}$ (dark blue) to $100 \mu \mathrm{M}$ (aqua). Error bars represent S.D. $(n=3)$.
Table 1 Photophysical properties of $\mathrm{Tb}^{3+}$ complexes

\begin{tabular}{lllll}
\hline Probe & Charge & $\tau(\mathrm{ms})$ & $K_{\mathrm{sv}}{ }^{a}\left(\mathrm{M}^{-1}\right)$ & $k_{\mathrm{q}}{ }^{a}\left(\mathrm{M}^{-1} \mathrm{~s}^{-1}\right)$ \\
\hline A1Tb & +3 & 1.52 & $1.9 \times 10^{5}$ & $1.2 \times 10^{8}$ \\
A2Tb & +1 & 1.96 & $4.0 \times 10^{4}$ & $2.1 \times 10^{7}$ \\
A3Tb & 0 & 1.87 & $1.7 \times 10^{4}$ & $9.1 \times 10^{6}$
\end{tabular}

${ }^{a} K_{\mathrm{sv}}$ and $k_{\mathrm{q}}$ were calculated from Stern-Volmer plots of luminescence lifetimes upon titration of NADH. Excitation wavelength was $340 \mathrm{~nm}$.

only moderate decreases in luminescence intensity and lifetime upon titration of NADH up to $100 \mu \mathrm{M}$ (Fig. S2 and S3, ESI $\dagger$ ).

We further investigated the photophysical properties of these $\mathrm{Tb}^{3+}$ complexes and the bimolecular quenching constants $\left(k_{\mathrm{q}}\right)$ upon titration of NADH. No significant differences were observed in the shapes of the absorption spectra and emission spectra, which may imply that the coordination structures of the three complexes are very similar (Fig. S4 and Table S2, ESI $\dagger$ ). As summarized in Table 1, the more positively charged complex was more sensitive to NADH. The trend was more clearly seen in quenching of A1Tb by NADPH, which possesses greater negative charge than NADH, and is therefore expected to have stronger electrostatic interaction than NADH with positively charged compounds (compare Tables S3 and S4, ESI $\dagger$ ). On the other hand, $100 \mu \mathrm{M} \mathrm{NAD}^{+}$ had little effect on luminescence intensity and no effect on lifetime (Table S5, ESI $\dagger$ ). We also measured the effect of several adenine-containing biological compounds, and showed that they do not cause dynamic quenching of the complex (Table S5, ESI $\dagger$ ). All these data suggest that electrostatic attraction between $\mathrm{Tb}^{3+}$ and phosphate groups, as well as reductive electron transfer or charge-transfer exciplex formation between $\mathrm{Tb}^{3+}$ and the reduced nicotinamide moiety at the excited state, is important for the dynamic quenching by $\mathrm{NAD}(\mathrm{P}) \mathrm{H}^{17}$ However, considering the energy diagram of $\mathrm{Tb}^{3+}$ complexes (Fig. S5, ESI $\dagger$ ), which starts with antenna excitation, followed by intersystem crossing and intramolecular energy transfer, and results in the excited state of $\mathrm{Tb}^{3+}$, it is possible that NAD(P)H also acts on the antenna moiety. Therefore, we further examined whether the excited state of the antenna is involved in the quenching process by direct excitation of the $\mathrm{Tb}^{3+}$ ion at $488 \mathrm{~nm}$ (Tables S3 and S4, ESI $\dagger$ ). The obtained quenching constants were the same for direct excitation $(488 \mathrm{~nm})$ and antenna excitation $(340 \mathrm{~nm})$, supporting the idea that $\mathrm{NAD}(\mathrm{P}) \mathrm{H}$ only acts in the excited state of $\mathrm{Tb}^{3+}$. In accordance with this view, the fluorescence of the antenna moiety, 2-Me azaxanthone, was hardly quenched in the presence of $10 \mu \mathrm{M}$ NADH (Fig. S6, $\mathrm{ESI}+$ ). Importantly, the $k_{\mathrm{q}}$ value of $\mathrm{A} 1 \mathrm{~Tb}$ for $\mathrm{NAD}(\mathrm{P}) \mathrm{H}$ (over $1 \times$ $10^{8} \mathrm{M}^{-1} \mathrm{~s}^{-1}$ ) was much larger than those for GSH and ascorbate, so A1Tb is tolerant of these reductive reagents, which are often used in enzyme assay (Table S1, ESI $\dagger$ ).

Next, we tested whether this quenching can be used to monitor enzyme reactions, because A1Tb responds to 1-100 $\mu \mathrm{M}$ $\mathrm{NAD}(\mathrm{P}) \mathrm{H}$, which is the optimal range for applications such as HTS. The selected model enzyme, lactate dehydrogenase (LDH, EC 1.1.1.27), is a NADH-dependent enzyme that catalyzes the reduction of pyruvate to $\mathrm{L}$-lactate while converting NADH into 
a
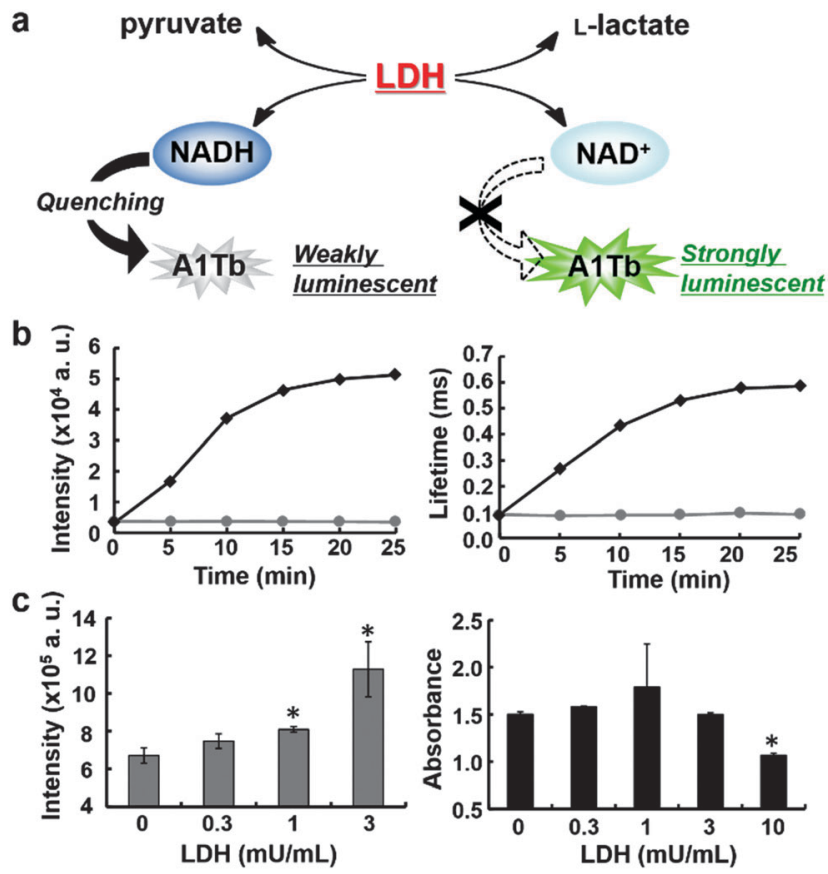

Fig. 3 (a) Schematic illustration of LDH assay using lanthanide luminescence. (b) Time course of luminescence intensity (left) or lifetime (right) of $4 \mu \mathrm{M} \mathrm{A1Tb}$ in the presence (black diamond) and absence (gray circle) of $\mathrm{LDH}\left(50 \mathrm{mU} \mathrm{mL} \mathrm{L}^{-1}\right.$ ). Initial concentrations of pyruvate and $\mathrm{NADH}$ were $125 \mu \mathrm{g} \mathrm{mL}^{-1}$ and $100 \mu \mathrm{M}$, respectively. (c) Comparison of time-resolved luminescence (left) and NADH absorption (right) measurements on microplates after incubation for 25 minutes. Error bars represent S.D. $(n=3)$. Asterisks indicate $p<0.05 \mathrm{vs}$. $0 \mathrm{mU} \mathrm{mL}^{-1}$

$\mathrm{NAD}^{+}$(Fig. 3a); it plays a key role in the anaerobic pathway of glucose metabolism. ${ }^{1}$ Because cancer cells tend to use this pathway instead of the aerobic pathway (Warburg effect), a specific subtype of this enzyme, LDH-A, is considered as an attractive target for anticancer drugs. ${ }^{1}$ The results of LDH assay with A1Tb in a cuvette are shown in Fig. 3b. Addition of LDH was accompanied by a monotonic increase in both luminescence intensity and lifetime of $\mathrm{A} 1 \mathrm{~Tb}$, while no change was observed in the absence of the enzyme. Unaltered LDH activity in the presence of A1Tb was confirmed by measuring NADH absorption (Fig. S7, ESI $\dagger$ ), which indicates that the probe does not interfere with the reaction of LDH. Also, the reaction products, L-lactate and $\mathrm{NAD}^{+}$, had negligible effect on the luminescence intensity or lifetime of A1Tb, at least up to $100 \mu \mathrm{M}$ (Table S5 and Fig. S8, ESI $\dagger$ ). These data demonstrate that the conversion of NADH into NAD ${ }^{+}$by LDH changed the luminescence properties of A1Tb. We next measured LDH activities on 96-well plates to see whether the method is suitable for HTS. As little as $1 \mathrm{mU} \mathrm{mL}^{-1}$ of LDH could be detected using A1Tb on microplates after incubation for 25 minutes. In contrast, $10 \mathrm{mU} \mathrm{mL}^{-1}$ was the lowest concentration that could be detected by absorption measurement of NADH at $340 \mathrm{~nm}$ (Fig. 3c). Thus, A1Tb provides a more sensitive measurement of enzyme activity than this classical method.

The scope of A1Tb is not limited to LDH. To demonstrate its generality, we performed preliminary time-resolved luminescence measurements for alcohol dehydrogenase (ADH, EC 1.1.1.1) and malate dehydrogenase (MDH, EC 1.1.1.37), and confirmed that $\mathrm{A} 1 \mathrm{~Tb}$ could also probe their activity on microplates with small S.D. values (Fig. S9 and S10, ESI†). In principle, our probe can monitor the activities of hundreds of NAD(P)H-dependent enzymes, and the number could be further increased by coupling these enzymes to other enzymes. A further advantage of our probe is that it is reversible (i.e. the luminescence can be turned on and off repeatedly). For example, when the NADH concentration in the sample was initially increased and then decreased, the probe could readily report these changes in real time (Fig. S11, ESI $\dagger$ ). This is in sharp contrast to reaction-based fluorescence and luminescence sensors. ${ }^{5}$

In conclusion, we have developed a novel assay platform for $\mathrm{NAD}(\mathrm{P}) \mathrm{H}$-dependent enzymes using luminescent $\mathrm{Tb}^{3+} \mathrm{com}^{-}$ plexes. Though many luminescent probes for biological molecules have been reported, ${ }^{7-9,12}$ most of them are specific to limited analytes and do not have practical utility for general assays. In contrast, probes for coenzymes ${ }^{13}$ including $\mathrm{NAD}(\mathrm{P}) \mathrm{H}$ are expected to have high generality. We believe that this is the first report to describe the interaction between $\mathrm{NAD}(\mathrm{P}) \mathrm{H}$ and luminescent lanthanide complexes. ${ }^{17}$ We showed that $\mathrm{NAD}(\mathrm{P}) \mathrm{H}$ dynamically quenches the luminescence of $\mathrm{Tb}^{3+}$ complexes, and this phenomenon can be used to monitor the activities of NAD(P)H-dependent enzymes. Although it is not suitable for imaging cellular NADH, our probe can monitor enzyme reactions in real time with good sensitivity. In this communication, we have focused on optimizing the net charge of the complexes, and the influence of other factors still needs to be studied to elucidate the quenching mechanism in more detail. We are currently synthesizing $\mathrm{Tb}^{3+}$ complexes with other antenna or chelator moieties to optimize sensitivity, and we aim to apply them for inhibitor screening and clinical diagnostic assays of NAD(P)H-dependent enzymes.

This work was supported by KAKENHI (Grant 22000006 to T.N., 24689003 and 24659042 to K.H., 24655147 to K.T., 25104506 to T.T., and 23249004 to Y.U.), by SENTAN, JST (K.H.), Grant-inAid for JSPS Fellows (H.I.) and by Astellas Foundation for Research on Metabolic Disorders (T.T.).

\section{Notes and references}

1 M. G. Vander Heiden, Nat. Rev. Drug Discovery, 2011, 10, 671.

2 (a) K. Smolková and P. Ježek, Int. J. Cell Biol., 2012, 273947; (b) M. K. Go, W. C. Zhang, B. Lim and W. S. Yew, Biochemistry, 2014, 53, 947.

3 S. L. Upstone, Encyclopedia of Analytical Chemistry, John Wiley \& Sons Ltd, Chichester, 2000.

4 M. J. Vázquez, S. Ashman, F. Ramón, D. Calvo, A. Bardera, J. J. Martín, M. Rüdiger, D. Tew and J. M. Domínguez, J. Biomol. Screening, 2006, 11, 75 .

5 H. Komatsu, Y. Shindo, K. Oka, J. P. Hill and K. Ariga, Angew. Chem., Int. Ed., 2014, 53, 3993.

6 (a) J. Yuan and G. Wang, Trends Anal. Chem., 2006, 25, 490; (b) I. Hemmilä, J. Biomol. Screening, 1999, 4, 303; (c) F. Degorce, A. Card, S. Soh, E. Trinquet, G. P. Knapik and B. Xie, Curr. Chem. Genomics, 2009, 3, 22.

7 (a) K. Hanaoka, K. Kikuchi, H. Kojima, Y. Urano and T. Nagano, J. Am. Chem. Soc., 2004, 126, 12470; (b) D. G. Smith, B. K. McMahon, R. Pal and D. Parker, Chem. Commun., 2012, 48, 8520. 
8 (a) M. S. Tremblay, M. Halim and D. Sames, J. Am. Chem. Soc., 2007, 129, 7570; (b) T. Terai, H. Ito, K. Kikuchi and T. Nagano, Chem. - Eur. J., 2012, 18, 7377; (c) T. Terai, K. Kikuchi, S. Iwasawa, T. Kawabe, Y. Hirata, Y. Urano and T. Nagano, J. Am. Chem. Soc., 2006, 128, 6938.

9 For review, see J.-C. G. Bünzli, Chem. Rev., 2010, 110, 2729.

10 R. A. Poole, C. P. Montgomery, E. J. New, A. Congreve, D. Parker and M. Botta, Org. Biomol. Chem., 2007, 5, 2055.

11 (a) F. Kielar, C. P. Montgomery, E. J. New, D. Parker, R. A. Poole, S. L. Richardson and P. A. Stenson, Org. Biomol. Chem., 2007, 5, 2975; (b) G.-L. Law, D. Parker, S. L. Richardson and K.-L. Wong, Dalton Trans., 2009, 8481.

12 R. A. Poole, F. Kielar, S. L. Richardson, P. A. Stenson and D. Parker, Chem. Commun., 2006, 4084.
13 (a) S. Nadella, P. M. Selvakumar, E. Suresh, P. S. Subramanian, M. Albrecht, M. Giese and R. Fröhlich, Chem. - Eur. J., 2012, 18, 16784; (b) E. A. Weitz, J. Y. Chang, A. H. Rosenfield and V. C. Pierre, J. Am. Chem. Soc., 2012, 134, 16099.

$14 \mathrm{X}$. Zhu and S. Z. Lever, Electrophoresis, 2002, 23, 1348.

15 J. Yu, D. Parker, R. Pal, R. A. Poole and M. J. Cann, J. Am. Chem. Soc., 2006, 128, 2294.

16 Replacing $\mathrm{Tb}^{3+}$ with $\mathrm{Eu}^{3+}$ greatly reduced the extent of quenching, as has been reported for other reductants. ${ }^{10,11 a}$

17 There is a report on the ground state reduction of the $\mathrm{Dy}^{3+}$ complex by NADH on the film (T. Basova, I. Jushina, A. G. Gürek, V. Ahsen and A. K. Ray, J. R. Soc., Interface, 2008, 5, 801), but we do not believe this is directly relevant to our probes, because our probes showed no change in absorption spectra. 